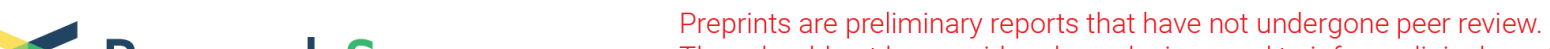 Research Square
or referenced by the media as validated information.
}

\section{In-Depth Biological Alteration Analysis of Plasmodium Knowelsilnfected Red Blood Cells Using A Non-Invasive Optical Imaging Technique}

\section{Egy Rahman Firdaus}

Kangwon National University College of Medicine: Kangwon National University School of Medicine Fauzi Muh

Kangwon National University College of Medicine: Kangwon National University School of Medicine Ji-Hoon Park

Kangwon National University College of Medicine: Kangwon National University School of Medicine

\section{Seong-Kyun Lee}

Kangwon National University College of Medicine: Kangwon National University School of Medicine

\section{Sung-Hun $\mathrm{Na}$}

Kangwon National University College of Medicine: Kangwon National University School of Medicine

\section{Won-Sun Park}

Kangwon National University College of Medicine: Kangwon National University School of Medicine Jin-Hee Han

Kangwon National University College of Medicine: Kangwon National University School of Medicine

\section{Kwon-Soo Ha}

Kangwon National University School of Medicine

EUN-TAEK HAN ( $\nabla$ etaekhan@gmail.com )

Kangwon National University School of Medicine https://orcid.org/0000-0003-1962-7199

\section{Research}

Keywords: Imaging technique, Tomography, P. knowlesi, hemoglobin.

Posted Date: July 20th, 2021

DOl: https://doi.org/10.21203/rs.3.rs-717357/v1

License: (c) (1) This work is licensed under a Creative Commons Attribution 4.0 International License. Read Full License 


\section{Abstract \\ Background}

Imaging techniques are commonly used for understanding disease mechanisms and their biological features in such a microenvironment of the cell. In recent work, the understanding of parasite biology of malaria Plasmodium knowlesi was compiled from functional in vitro and imaging analysis using serial block-face scanning electron microscopy (SEM). Sample fixation during SEM analysis possibly makes the parasite membrane destruction.

\section{Methods}

In this study, we used a non-invasive optical technique, tomography, to explore the biological changes during the development of infected red blood cells (RBCs) by P. knowlesi. The commercial common-path diffraction optical tomography was used to measure individual infected RBCs' three-dimensional refractive index tomogram without an additional dyeing agent.

\section{Results}

We simultaneously examined structural, chemical, and physical modification by a parasite inside of host RBCs. The results had similar patterns with previous studies using SEM and other parasites such as $P$. falciparum and Babesia. Interestingly we found that $P$. knowlesi did not use hemoglobin as much as $P$. falciparum as a source of nutrition.

\section{Conclusions}

This tomography technique opens new ways to study the pathophysiology of malaria parasites more efficiently and is considered a promising tool for studying each Plasmodium species more in-depth.

\section{Background}

Malaria pathophysiology is still an interesting topic to be explored. A variety of Plasmodium species with an extensive variant of each feature makes malaria still hard to be eradicated. In addition, immune evasion and drug resistance to malaria have been touched by multidisciplinary expertise [1-3]. Imaging techniques have been used for a long time in malaria pathophysiology studies. It has been continuously developed since the discovery of classical light microscope to exceptionally sophisticated technology such as Förster resonance energy transfer (FRET), fluorescence lifetime imaging microscopy (FLIM), third-harmonic generation (THG), two-photon absorption fluorescence (2PAF) imaging, and common-path diffraction optical tomography (cDOT) [4]. 
Tomography, one of the non-invasive optical techniques, has been used to explore the biological changes during the development of $P$. falciparum and Babesia parasites-infected red blood cells (RBCs) $[5,6]$. The tomography principle uses optical interferometry or digital holography, which is non-invasive, label-free, and quantitative. In addition, CDOT is a recent technique that can measure both the three-dimensional structure and dynamics of live cells simultaneously $[6,7]$.

Out of 6 Plasmodium species that infect humans, P. knowlesi is considered to be a threat pathogen. It is recognized as zoonotic malaria and has started to infect humans, particularly in Malaysia, and spread out to other areas in Southeast Asia. Besides, $P$. knowlesi also showed a symptom like cerebral malaria as found in $P$. falciparum $[8,9]$. During the host cell invasion, the parasite modifies the host cell environment to support their life, leads to morphological, biochemical, and mechanical modification [10, $11]$.

Recently $P$. knowlesi topography study applied for structural observation using serial block-face scanning electron microscopy [12]. However, that technique required a particular treatment like fixation before analysis. In addition, it has a limitation in determining live dynamics of parasite and host cells because of the fixation and metal coating steps $[6,13,14]$. Instead of using an additional treatment, our study focuses on live cells for biological analysis. Thus, the cells are kept in the natural form without losing a parasite membrane during observation. Tomography is considered an easy and fast technique for individual cell qualitative and quantitative analyses.

This investigation used $\mathrm{CDOT}$ of individual $P$. knowlesi-infected RBCs to investigate the alteration of morphological, biochemical, and mechanical parameters in every asexual developmental stage parasites. This research has shown that all parameters could be quantified as described in our data, including volume, surface area, sphericity, hemoglobin content, and membrane fluctuation. We also compared the data to $P$. falciparum as the most malignant human malaria parasites. From all data, we found that the hemoglobin consumption of $P$. knowlesi was less than $P$. falciparum as a source of nutrition. In the end, this method provides an extremely simple and accessible for studying malaria pathophysiology.

\section{Methods}

\section{Parasite culture}

P. knowlesi A1.H1 adapted human RBCs was cultured in RPMI 1640 media (Invitrogen Life Technologies, Grand Island, NY) supplemented by horse serum (Gibco, Life Technologies), L-glutamine, 25 mM HEPES (Invitrogen Life Technologies), and 0.5\% Albumax II (Invitrogen Life Technologies). The culture was maintained at $2 \%$ hematocrit with mixed gas $\left(90 \% \mathrm{~N}_{2}, 5 \% \mathrm{CO}_{2}\right.$, and $\left.5 \% \mathrm{O}_{2}\right)$ at $37^{\circ} \mathrm{C}$ while $P$. falciparum was cultured with slightly different supplementation as described elsewhere [15]. Both parasites were treated with gentamicin to prevent contamination.

\section{Schizont synchronization}


The synchronization of parasite stages was conducted by setting a schizont as an initial stage. $P$. knowlesi schizonts were isolated using $50 \%$ nycodenz, while $P$. falciparum used magnetic-activated cell sorting (MACS) system [16]. Purified schizont was re-culture at $2 \%$ parasitemia, the ring stage of $P$. knowlesi was observed $10 \mathrm{hrs}$ after the invasion, while P.falciparum needed $18 \mathrm{hrs}[17,18]$.

\section{Analysis of parasite-infected human RBCs alteration}

Three to five percent parasitemia of each parasite stage was set for further analysis using a commercial 3D cDOT system (HT-1H, Tomocube Inc., Republic Korea). The parasite was diluted with Dulbecco's phosphate-buffered saline (DPBS) without calcium and magnesium, then apply to $25^{\prime} 50 \mathrm{~mm}$ coverslip (Matsunami Glass Ind., Osaka, Japan). The data was visualized using Tomo Studio software (Tomocube Inc., Daejeon, Republic Korea) and analyzed quantitatively by RBCs characterization software using a specific algorithm [19]. Fifteen samples of each stage were used for further analysis.

\section{Statistical analysis}

GraphPad Prism (GraphPad Sofware, San Diego, CA) was used to analyze data statistically. The student's $t$-test was applied to determine differences between the two group data. The data with $p<0.05$ were considered to have significant differences.

\section{Result}

\section{Three-dimensional refractive index maps of malaria- infected RBCs}

Data of refractive index ( $\mathrm{RI})$ generated a 3-dimensional optical tomogram that was utilized for analyzing various inside and outside features of cells [20]. The specific developmental blood stage of the malaria parasite was observed after synchronization (Fig. 1). The RI of the healthy RBC following maturation of $P$. knowlesi parasite stage was gradually decreased with their mean values for healthy RBCs $(1.375 \pm$ $0.005)$, ring (1.370 \pm 0.004$)$, trophozoite $(1.369 \pm 0.004)$, and schizont $(1.367 \pm 0.004)$ stages. The value could be more understandable by comparing $\mathrm{RI}$ and phase map images of each developmental stage (Fig. 2). Healthy RBCs are described as donuts shapes due to their biconcave shape. The dark red color showed the highest value, meanwhile, the yellow color showed the lowest RI. The uniformity of healthy RBCs content was described as uniform color, most of the RBCs containing $95 \%$ spherical protein like hemoglobin [21]. Whereas the parasitized RBCs seen have a gradient color affected by parasite-filled RBCs space. The yellow color exhibited parasite cytoplasm, while the dark red indicated hemozoin. The pattern also was observed in $P$. falciparum $\mathrm{RI}$ with their values for ring $(1.373 \pm 0.004)$, trophozoite $(1.371$ $\pm 0.003)$, and schizont $(1.367 \pm 0.004)$ stages.

Morphological alteration of P. knowlesi-RBCs in different stages 
During infection, the parasite growth induced host cell constituents deficiency [22]. It is reflected by morphological changes of infected RBCs, including the RBCs shape that was becoming more spherical from the initial biconcave or donuts shape [23]. Decreasing cellular volume and surface area of the RBCs also were observed in the infected RBCs. The shape alteration of infected-RBCs showed in the mean value of sphericity which gradually increased from healthy $(0.616 \pm 0.103)$, ring $(0.723 \pm 0.090)$, trophozoite $(0.721 \pm 0.099)$, and schizont $(0.770 \pm 0.041)$ (Fig. $3 \mathrm{~A})$ stages. In terms of mean values of cytosol volume for healthy RBCs $(86.686 \pm 7.619 \mathrm{fL})$, ring $(80.413 \pm 9.331 \mathrm{fL})$, trophozoite $(76.084 \pm 8.282$ $\mathrm{fL}$ ), and schizont (71.177 $\pm 11.490 \mathrm{fL}$ ) stages (Fig. 3C). Decreasing that value was caused by the parasite occupied and digesting the nutrition provided in the RBCs cytosol. With the increasing of RBCs sphericity during parasite maturation, the surface area of the RBCs continuously decreased for healthy RBCs $\left(154.863 \pm 16.924 \mathrm{um}^{2}\right)$, ring $\left(150.802 \pm 12.270 \mathrm{um}^{2}\right)$, trophozoite $\left(150.009 \pm 16.916 \mathrm{um}^{2}\right)$, and schizont $\left(142.176 \pm 23.840 \mathrm{um}^{2}\right.$ ) stages (Fig. 3D). A similar pattern of cellular volume and surface area of $P$. falciparum also could observe as following data $76.632 \pm 18.553,71.075 \pm 17.240,63.395 \pm 15.388 \mathrm{fL}$ (cellular volume), and 76.632 $\pm 18.553,71.075 \pm 17.240,63.395 \pm 15.388$ um$^{2}$ (surface area) for healthy RBCs, ring, trophozoite, and schizont stages, respectively.

\section{Alteration of biological dynamics in asexual stages of P. knowlesi-infected RBCs}

Parasite actively proliferates, and growth utilizes an environment inside RBCs. It affects an alteration of the structural and live dynamics of host RBCs [24]. RBC membrane fluctuation was used to identify live dynamics alteration during infection. The data showed that increasing the mean value of fluctuation due to maturation of parasite for healthy RBCs $(78.330 \pm 23.051 \mathrm{~nm})$, ring $(114.634 \pm 31.060 \mathrm{~nm})$, and schizont $(237.643 \pm 266.212 \mathrm{~nm})$, however, the trophozoite $(107.395 \pm 35.443 \mathrm{~nm})$ slightly decreases (Fig. 4A).

\section{Hemoglobin Content In Each Stage Of Parasite-infected Rbcs}

The implication of a significant decrease of cytosol volume in the $P$. knowlesi-infected RBCs was obviously shown in decreasing hemoglobin content following the maturation of the parasite. Hemoglobin is a major content of cytoplasm, and it was used by the parasite as the nutrient for their growth and proliferation $[25,26]$. The more merozoite exists inside RBCs, and the more hemoglobin will decrease. The mean value of hemoglobin content was observed from the healthy $(29.143 \pm 4.103 \mathrm{pg})$, ring $(24.623 \pm$ $3.154 \mathrm{pg})$, trophozoite $(23.446 \pm 2.913 \mathrm{pg})$, and schizont $(22.003 \pm 3.913 \mathrm{pg})$ stages (Fig. 4B). However, $P$. falciparum-infected RBCs were calculated in the ring (19.203 $\pm 4.666 \mathrm{pg})$, trophozoite $(16.941 \pm 4.137 \mathrm{pg})$, and schizont $(14.597 \pm 3.541 \mathrm{pg})$ stages, which reflects $P$. falciparum more actively consumed hemoglobin than $P$. knowlesi.

\section{Discussion}


Once malaria parasites entered and started to take over the RBCs, their morphology is actively changed due to their growth and development. Malaria parasites transform during the maturation from immature ring and trophozoite to mature schizont stages. The environment within the host RBCs that majorly consist of protein cytoplasm or hemoglobin will decrease as well as parasites use it for their metabolism [27]. In addition, the loss of the donuts shape causes a decrease in the surface area of red blood cells and makes their shape more spherical, which affects the loss of deformability. [23, 28].

The parasites use hemoglobin as a precursor of amino acid synthesis [29]. Hemoglobin is degraded to the toxic product called hematin then converts to a structural compound called hemozoin to avoid the reactive oxygen species [30,31]. Hemozoin produces high contrast imaging; some microscopy was observed, such as polarization microscopy, dark-field microscopy, and resonance Raman microscopy [4, $32,33]$. The schizont refractive index also exhibited it was significantly increased because of hemozoin production. It is also affected by many proteins released out during merozoite egress [34].

A major protein released during the schizont stage has a photolytic function such as cytoskeletondegrading malarial proteases, falcipain-2 and plasmepsin II, plus a family of putative papain-like proteases called serine repeat antigen [34, 35]. It gave a consequence to have a high fluctuation in the RBCs membrane. Besides, several excess nutrients are transferred by several pathways like new permeability pathways studied by several groups, especially in $P$. falciparum [36]. Meanwhile, the ring stage has a more considerable fluctuation value, probably because it still requires an arrangement of a new host RBC environment.

Our study focused on the RBCs as an object, while previous research focused on the parasite alteration itself [12]. This study also comparable with previous research that used a similar technique in $P$. falciparum and Babesia parasites. Our study found that the uniqueness of $P$. knowlesi tended not to make the significant alteration within-host RBCs compare to $P$. falciparum in hemoglobin content. The fact that hemoglobin content of $P$. knowlesi-infected RBCs was higher than $P$. falciparum, might occur with the following assumptions: (1) P. knowlesi life cycle shorter than $P$. falciparum; thus, $P$. knowlesi did not have enough time to consume the hemoglobin. (2) Host preference differences. $P$. knowlesi is used to stay in the monkey RBCs but should use human RBCs may affect parasite metabolism. (3) The parasite might use another source more than hemoglobin. However, those hypotheses needed further study.

The level of cytosol volume of $P$. falciparum-infected RBCs was more drastically decreased than $P$. knowlesi. The clinical data has strengthened this assumption where P.knowlesi patients hemoglobin amount was higher than P.falciparum with the following described for $P$. knowlesi $(9.7 \mathrm{~g} / \mathrm{dL})$ and $P$. falciparum $(7.15 \mathrm{~g} / \mathrm{dL})$ [37]. This study could be used in the understanding mechanism of parasites utilize the host environment. In addition, our finding leads to a possibility that $P$. knowlesi treatment against hemoglobin metabolism may not affect much more than $P$. falciparum.

\section{Conclusion}


In conclusion, the application of CODT opens a new way that much easier to study the pathophysiology of malaria by exploiting the live sample using the refractive index. This study supports that each Plasmodium species has a particular profile in utilizing host resources that correlate to different pathways of those species carry on their lives.

\section{Abbreviations}

SEM

Scanning electron microscopy; RBCs:Red blood cells, FRET:Förster resonance energy transfer, FLIM:Fluorescence lifetime imaging microscopy, THG:Third-harmonic generation (THG); 2PAF:Twophoton absorption fluorescence; cDOT:Common-path diffraction optical tomography; $P$. falciparum: Plasmodium falciparum; P. knowlesi:Plasmodium knowlesi; MACS:Magnetic-activated cell sorting; DPBS:Dulbecco's phosphate-buffered saline; RI:refractive index.

\section{Declarations}

\section{Ethics approval and consent to participate}

This study was approved by the Ethics Committee, Kangwon National University Hospital, ROK (IRB No. 2014-08-008-002). Informed consent was obtained from all of the participants

\section{Consent for publication}

Not applicable.

\section{Competing interest}

The authors declare that they have no competing interests.

\section{Funding}

This study was supported by a grant from the National Research Foundation of Korea (NRF) grant funded by the Korean government (MSIP)(NRF-2021R1A2C2008235) and by Basic Science Research Program through the National Research Foundation of Korea (NRF) funded by the Ministry of Science, ICT and Future Planning (NRF-R1A4A1031574), by a grant from the Korea Association of Health Promotion (2019-02) and by 2018 Research Grant (PoINT) from Kangwon National University.

\section{Author contributions}

E.R.F and E.-T. H. designed the study. E.R.F, F.M, J.-H P., and S.-K. L. conducted the laboratory work and data analysis. S.-H. N., W.-S. P., J.-H. H., and K.-W. H. supported materials and data analysis. All authors contributed to writing, reading, and approving the manuscript.

\section{Availability of data and material}


The datasets supporting the conclusions are included in the article.

\section{Author details}

${ }^{1}$ Department of Medical Environmental Biology and Tropical Medicine, Kangwon National University School of Medicine, Chuncheon, Gangwon-do 24341, Republic of Korea

${ }^{2}$ Department of Obstetrics and Gynecology, Kangwon National University School of Medicine, Chuncheon, Gangwon-do 24341, Republic of Korea

${ }^{3}$ Department of Physiology, School of Medicine, Kangwon National University, Chuncheon, Gangwondo 24341, Republic of Korea

${ }^{4}$ Department of Molecular and Cellular Biochemistry, Kangwon National University School of Medicine, Chuncheon, Gangwon-do 24341, Republic of Korea

\section{References}

1. Miller LH, Ackerman HC, Su XZ, Wellems TE. Malaria biology and disease pathogenesis: insights for new treatments. Nat Med. 2013;19(2):156.

2. Rénia L, Goh YS. Malaria parasites: the great escape. Front Immunol. 2016;7:463.

3. Tanner M, Greenwood B, Whitty CJ, Ansah EK, Price RN, Dondorp AM, et al. Malaria eradication and elimination: views on how to translate a vision into reality. BMC Med. 2015;13(1):167.

4. Cho S, Kim S, Kim Y, Park Y. Optical imaging techniques for the study of malaria. Trends Biotechnol. 2012;30(2):71-9.

5. Park Y, Diez-Silva M, Popescu G, Lykotrafitis G, Choi W, Feld MS, et al. Refractive index maps and membrane dynamics of human red blood cells parasitized by Plasmodium falciparum. Proc Natl Acad Sci U S A. 2008;105(37):13730-5.

6. Park H, Hong SH, Kim K, Cho SH, Lee WJ, Kim Y, et al. Characterizations of individual mouse red blood cells parasitized by Babesia microti using 3-D holographic microscopy. Sci Rep. 2015;5:10827.

7. Kim YS, Lee S, Jung J, Shin S, Choi HG, Cha GH, et al. Combining Three-Dimensional Quantitative Phase Imaging and Fluorescence Microscopy for the Study of Cell Pathophysiology. Yale J Biol Med. 2018;91(3):267.

8. Singh B, Daneshvar C. Human infections and detection of Plasmodium knowlesi. Clin Microbiol Rev. 2013;26(2):165-84.

9. Cox-Singh J, Hiu J, Lucas SB, Divis PC, Zulkarnaen M, Chandran P, et al. Severe malaria - a case of fatal Plasmodium knowlesi infection with post-mortem findings: a case report. Malar J. 2010;9(1):10.

10. Kapczuk P, Kosik-Bogacka D, Łanocha-Arendarczyk N, Gutowska I, Kupnicka P, Chlubek D, et al. Selected Molecular Mechanisms Involved in the Parasite Host System Hymenolepis diminuta Rattus 
norvegicus. Int J Mol Sci. 2018;19(8):2435.

11. Chandramohanadas R, Park Y, Lui L, Li A, Quinn D, Liew K, et al. Biophysics of malarial parasite exit from infected erythrocytes. PLoS One. 2011;6(6):e20869.

12. Liu B, Blanch AJ, Namvar A, Carmo O, Tiash S, Andrew D, et al. Multimodal analysis of Plasmodium knowlesi-infected erythrocytes reveals large invaginations, swelling of the host cell, and rheological defects. Cell Microbiol. 2019;21(5):e13005.

13. Gohil S, Kats LM, Sturm A, Cooke BM. Recent insights into alteration of red blood cells by Babesia bovis: moovin' forward. Trends Parasitol. 2010;26(12):591-9.

14. Gluenz E, Wheeler RJ, Hughes L, Vaughan S. Scanning and three-dimensional electron microscopy methods for the study of Trypanosoma brucei and Leishmania mexicana flagella. Methods Cell Biol. 2015;127:509-42.

15. Firdaus ER, Park J-H, Muh F, Lee S-K, Han J-H, Lim C-S, et al. Performance Evaluation of Biozentech Malaria Scanner in Plasmodium knowlesi and P. falciparum as a New Diagnostic Tool. Korean J Parasitol. 2021;59(2):113-9.

16. Ribaut C, Berry A, Chevalley S, Reybier K, Morlais I, Parzy D, et al. Concentration and purification by magnetic separation of the erythrocytic stages of all human Plasmodium species. Malar J. 2008;7(1):45.

17. Ngernna S, Chim-Ong A, Roobsoong W, Sattabongkot J, Cui L, Nguitragool W. Efficient synchronization of Plasmodium knowlesi in vitro cultures using guanidine hydrochloride. Malar $\mathrm{J}$. 2019;18(1):148.

18. Verzier LH, Coyle R, Singh S, Sanderson T, Rayner JC. Plasmodium knowlesi as a model system for characterising Plasmodium vivax drug resistance candidate genes. PLoS Negl Trop Dis. 2019;13(6):e0007470.

19. Koo SE, Jang S, Park Y, Park CJ. Reconstructed Three-Dimensional Images and Parameters of Individual Erythrocytes Using Optical Diffraction Tomography Microscopy. Ann Lab Med. 2019;39(2):223-6.

20. Liu PY, Chin LK, Ser W, Chen HF, Hsieh CM, Lee CH, et al. Cell refractive index for cell biology and disease diagnosis: past, present and future. Lab Chip. 2016;16(4):634-44.

21. Longeville S, Stingaciu LR. Hemoglobin diffusion and the dynamics of oxygen capture by red blood cells. Sci Rep. 2017;7(1):10448.

22. Firdaus ER, Park JH, Lee SK, Park Y, Cha GH, Han ET. 3D morphological and biophysical changes in a single tachyzoite and its infected cells using three-dimensional quantitative phase imaging. $\mathrm{J}$ Biophotonics. 2020;13(8):e202000055.

23. Diez-Silva M, Dao M, Han J, Lim CT, Suresh S. Shape and Biomechanical Characteristics of Human Red Blood Cells in Health and Disease. MRS Bull. 2010;35(5):382-8.

24. Boulet C, Doerig CD, Carvalho TG. Manipulating Eryptosis of Human Red Blood Cells: A Novel Antimalarial Strategy? Front Cell Infect Microbiol. 2018;8:419. 
25. Kakhniashvili DG, Bulla LA Jr, Goodman SR. The human erythrocyte proteome: analysis by ion trap mass spectrometry. Mol Cell Proteomics. 2004;3(5):501-9.

26. Pishchany G, Skaar EP. Taste for blood: hemoglobin as a nutrient source for pathogens. PLoS Pathog. 2012;8(3):e1002535.

27. Goldberg DE, Slater AF, Cerami A, Henderson GB. Hemoglobin degradation in the malaria parasite Plasmodium falciparum: an ordered process in a unique organelle. Proc Natl Acad Sci U S A. 1990;87(8):2931-5.

28. Safeukui I, Buffet PA, Perrot S, Sauvanet A, Aussilhou B, Dokmak S, et al. Surface area loss and increased sphericity account for the splenic entrapment of subpopulations of Plasmodium falciparum ring-infected erythrocytes. PLoS One. 2013;8(3):e60150.

29. Soni R, Sharma D, Rai P, Sharma B, Bhatt TK. Signaling Strategies of Malaria Parasite for Its Survival, Proliferation, and Infection during Erythrocytic Stage. Front Immunol. 2017;8:349.

30. Fong KY, Wright DW. Hemozoin and antimalarial drug discovery. Future Med Chem. 2013;5(12):1437-50.

31. Aich A, Freundlich M, Vekilov PG. The free heme concentration in healthy human erythrocytes. Blood Cells Mol Dis. 2015;55(4):402-9.

32. Romagosa C, Menendez C, Ismail MR, Quintó L, Ferrer B, Alonso PL, et al. Polarisation microscopy increases the sensitivity of hemozoin and Plasmodium detection in the histological assessment of placental malaria. Acta Trop. 2004;90(3):277-84.

33. Jamjoom GA. Dark-field microscopy for detection of malaria in unstained blood films. J Clin Microbiol. 1983;17(5):717-21.

34. Blackman MJ. Malarial proteases and host cell egress: an 'emerging' cascade. Cell Microbiol. 2008;10(10):1925-34.

35. Collins CR, Hackett F, Atid J, Tan MSY, Blackman MJ. The Plasmodium falciparum pseudoprotease SERA5 regulates the kinetics and efficiency of malaria parasite egress from host erythrocytes. PLoS Pathog. 2017;13(7):e1006453.

36. Dickerman BK, Elsworth B, Cobbold SA, Nie CQ, McConville MJ, Crabb BS, et al. Identification of inhibitors that dually target the new permeability pathway and dihydroorotate dehydrogenase in the blood stage of Plasmodium falciparum. Sci Rep. 2016;6:37502.

37. Barber BE, William T, Jikal M, Jilip J, Dhararaj P, Menon J, et al. Plasmodium knowlesi malaria in children. Emerg Infect Dis. 2011;17(5):814.

\section{Figures}




\section{Mixed stage culture}
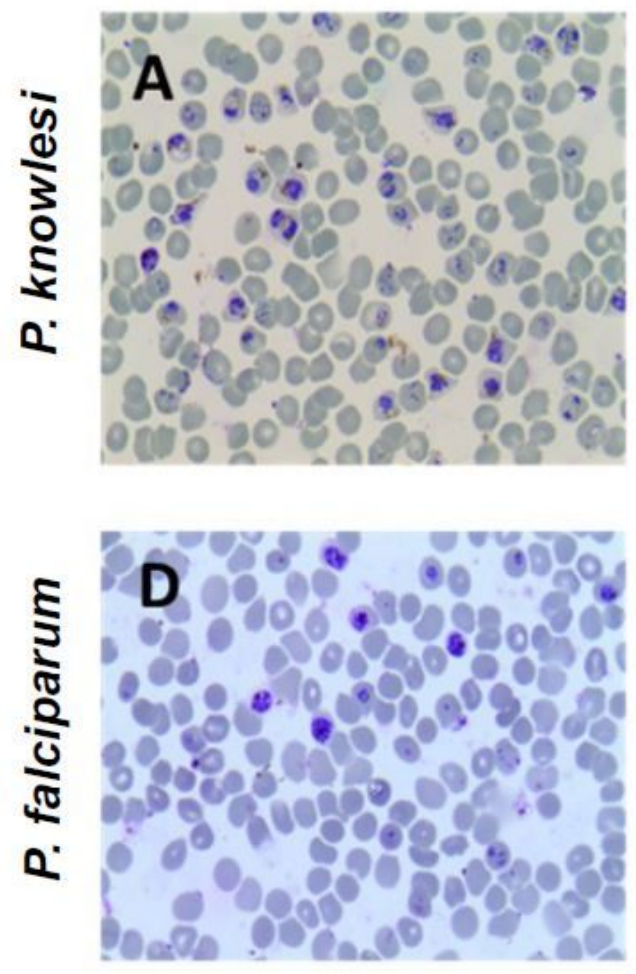

Synchronized schizont
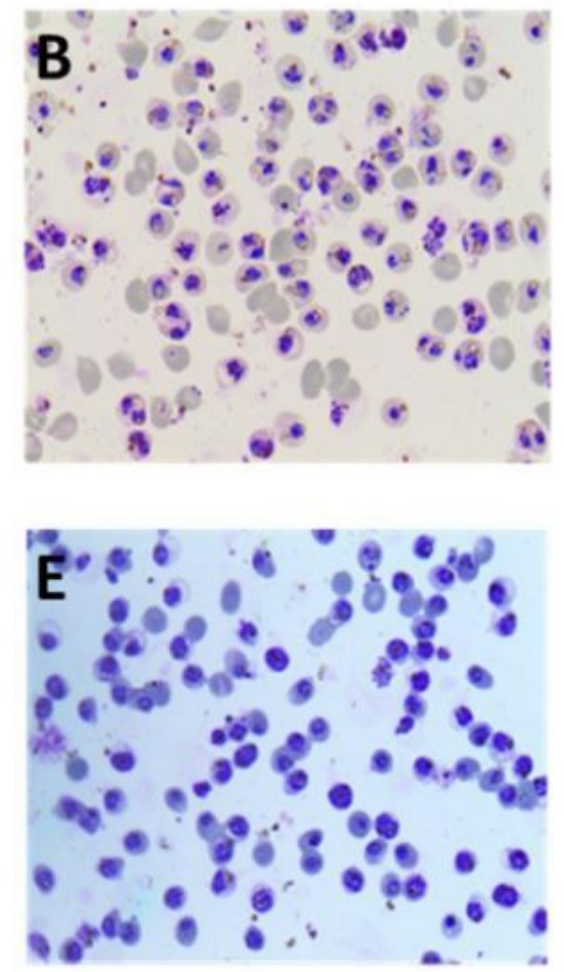

Ring stage after invasion
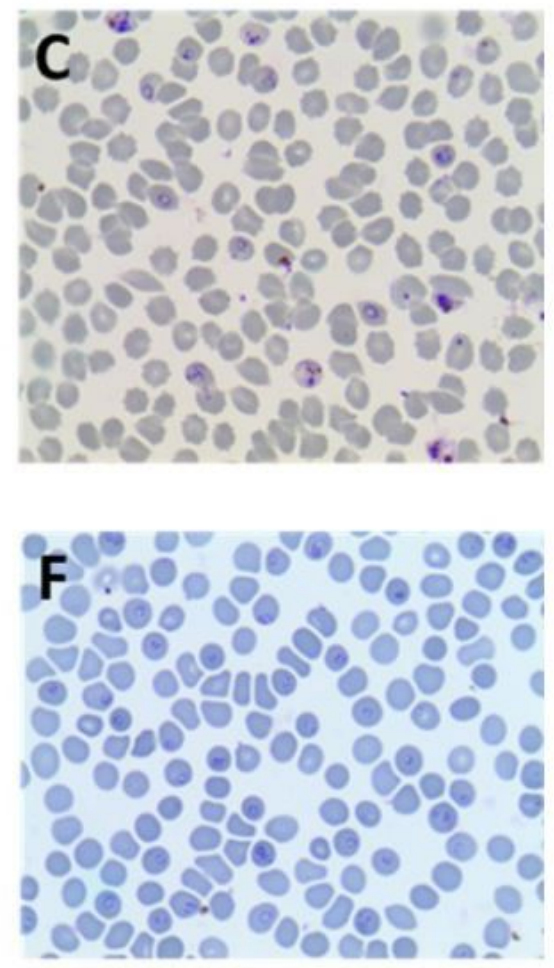

Fig. 1

Figure 1

Morphology of malaria parasite during synchronization. (A-C) Plasmodium knowlesi culture, (D-F) P. falciparum culture. ( $A$ and $D$ ) mixed stages of parasites, $(B$ and $E$ ) synchronized schizont stage, and (C and F) newly invaded ring stage. 


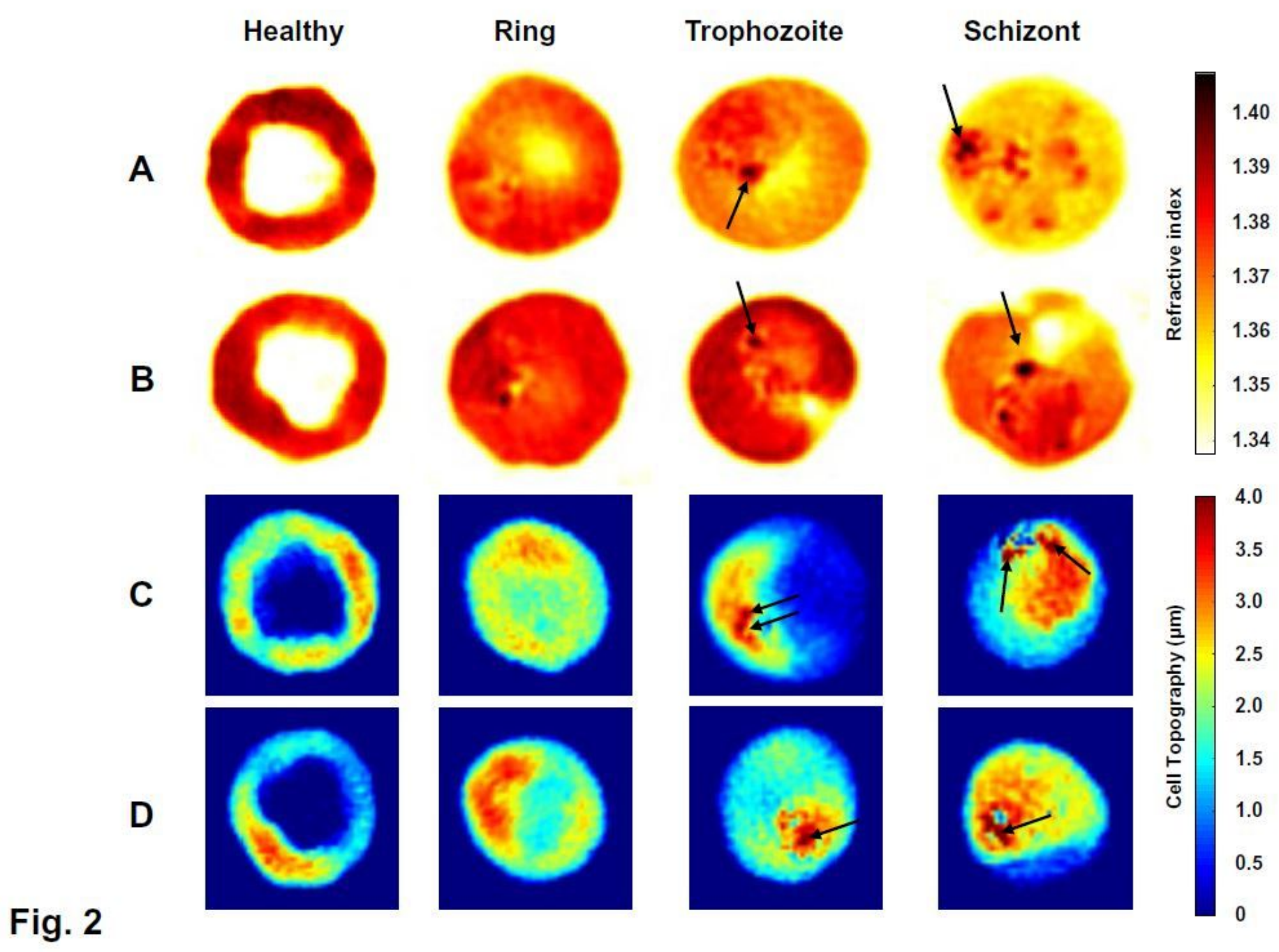

Figure 2

Three-dimensional refractive index and phase map of P. knowlesi-infected RBCs. (A-B) 3D RI maps of P. knowlesi-infected RBCs and (C-D) phase map image. The black arrow indicates hemozoin. 
A

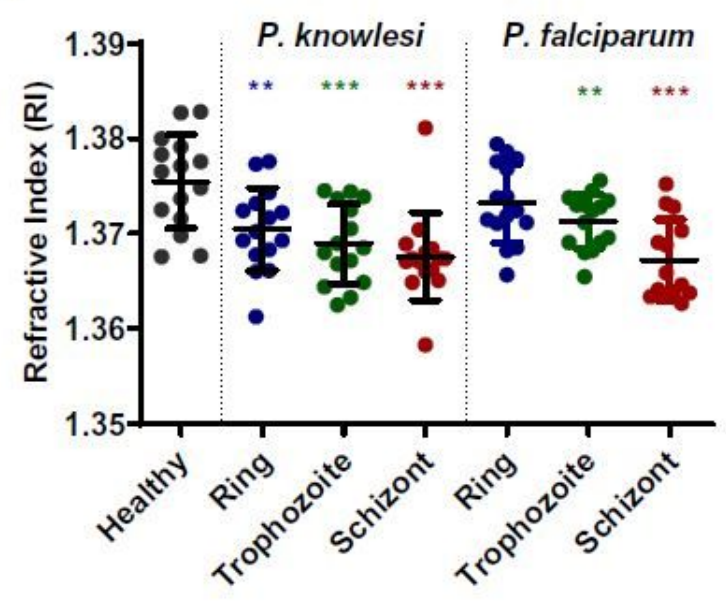

C

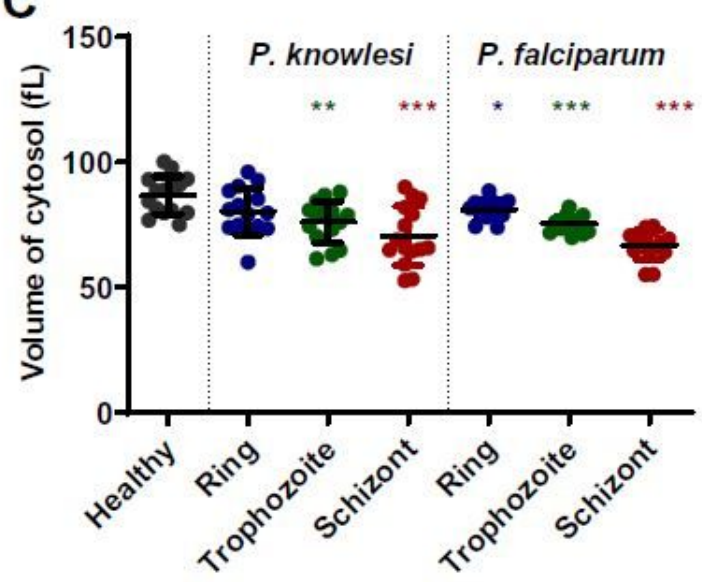

B

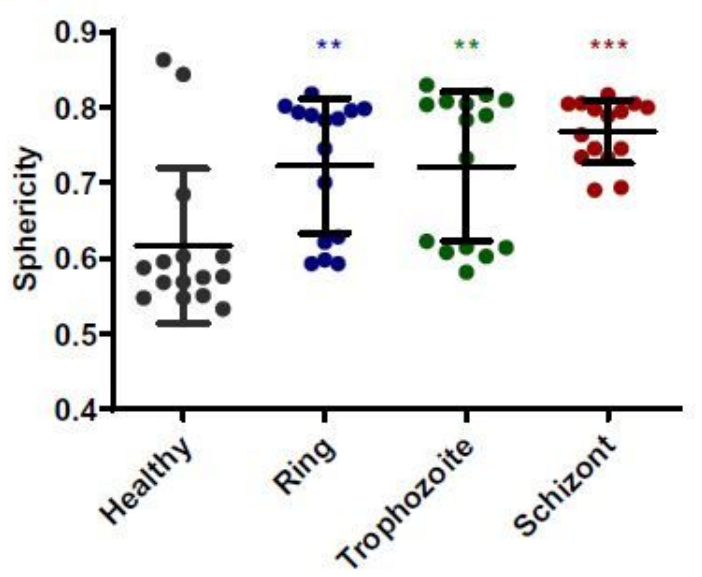

D

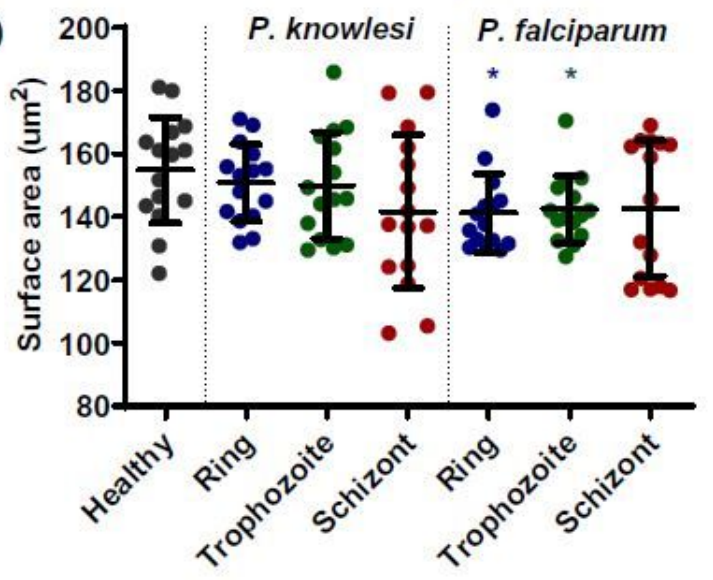

Fig. 3

Figure 3

Reflective index and morphological profile of P. knowlesi-infected RBCs. (A) refractive index, (B) sphericity, (C) cellular volume, and (D) surface area of P. knowelesi-infected RBCs. 

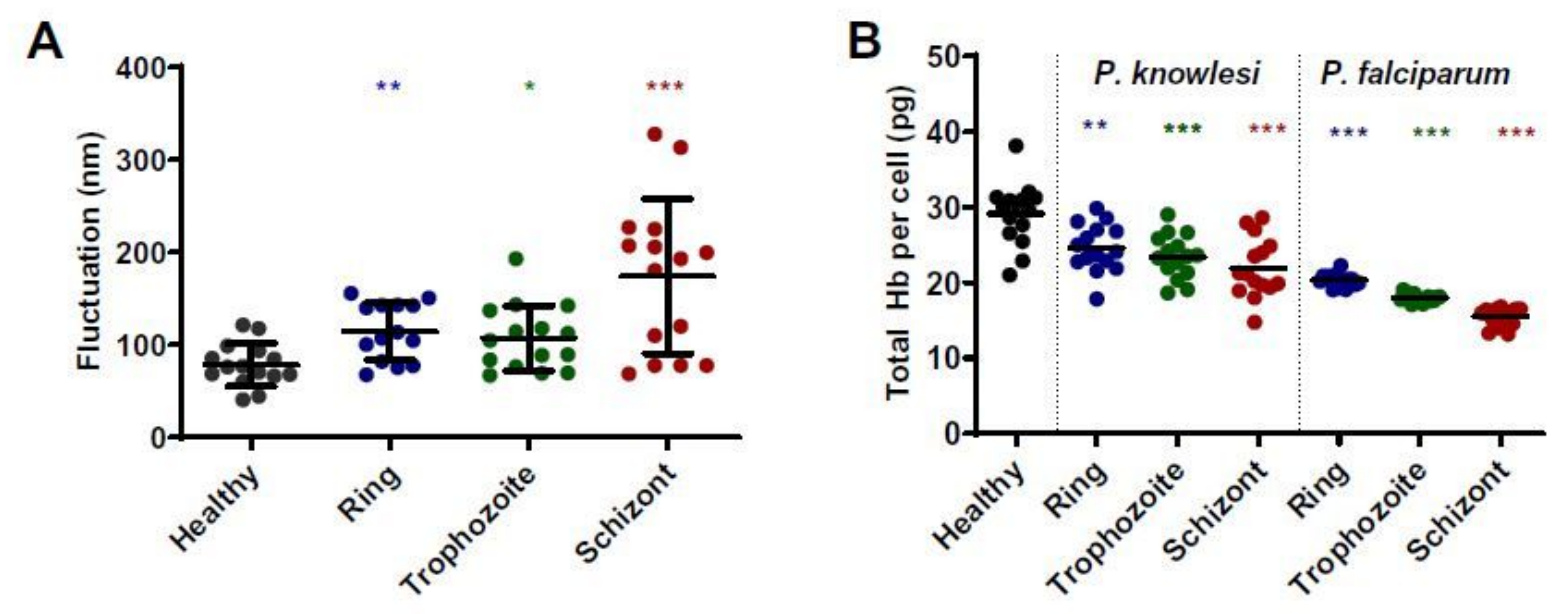

Fig. 4

\section{Figure 4}

Membrane dynamic and biochemical alteration of P. knowlesi and P. falciparum-infected RBCs. (A) Fluctuation of each stage in P. knowelesi-infected RBCs and (B) hemoglobin content both P. knowlesi- and P. falciparum-infected RBCs.

\section{Supplementary Files}

This is a list of supplementary files associated with this preprint. Click to download.

- GraphicalabstractSub.pdf 